\title{
Propofol effects in rodent models of traumatic brain injury: a systematic review
}

\author{
Riyadh Firdaus ${ }^{*}$, Sandy Theresia ${ }^{\oplus}$, Ryan Austin ${ }^{\oplus}$, Rani Tiara ${ }^{\odot}$
}

\begin{abstract}
Background: Traumatic brain injury (TBI) causes high mortality and disability worldwide. Animal models have been developed to explore the complex processes in TBI. Propofol is used to manage head injuries during surgical intervention and mechanical ventilation in patients with TBI. Many studies have investigated the neuroprotective effect of propofol on TBI. However, other studies have shown neurotoxic effects.

Objectives: To review systematically the literature regarding the neuroprotective and neurotoxic effects of propofol in rodent models of TBI.

Methods: Data from rodents as models of TBI with propofol as one of the intervention agents, and/or comparing the neuroprotective effects of propofol with the other substances in rodent models of TBI, were obtained from PubMed, EBSCO Host, and ProQuest databases. The PRISMA 2020 statement recommendations were followed and research questions were developed based on PICOS guidelines. Data was extracted from the literature using a standardized Cochrane method.

Results: We analyzed data from 12 articles on physiological changes of experimental animals before and after trauma, the effects of propofol administration, and the observed neurotoxic effects. The effects of propofol administration were observed in terms of changes in traumatic lesion volume, the release of antioxidants and inflammatory factors, and the neurological function of rodent models of TBI.

Conclusion: Propofol has neuroprotective and neurotoxic effects via several mechanisms, and various doses have been used in research to determine its effects. The timing of administration, the dose administered, and the duration of administration contribute to determine the effect of propofol in rodent models of TBI. However, the doses that produce neuroprotective and neurotoxic effects are not yet clear and further research is needed to determine them.
\end{abstract}

Keywords: anesthetics; brain injuries, traumatic; neuroprotection; propofol; Rodentia

Traumatic brain injury (TBI) causes high mortality and disability worldwide [1]. Data from 1990 to 2016 show 27 million TBI cases, with an average incidence of 369 cases per 100,000 population every year worldwide. This number might be lower than the actual number of cases as the incidence and distribution of TBI across nations remain unknown $[2,3]$. TBI is an injury to the brain that is usually caused by a bump, blow, or jolt to the head from blunt trauma or penetrating injury, such as from a motor vehicle accident or a gunshot [4]. TBI consists of primary and secondary injuries. Primary brain injury (focal and diffuse) results from mechanical injury at the time of the trauma, whereas secondary brain injury is caused by the physiological responses to the initial injury, leading to release of neurotoxic substances and neuronal cell death $[5,6]$. Hypotension and systemic hypoxia can exacerbate the secondary injury and are strongly associated with higher mortality risks

*Correspondence to: Riyadh Firdaus, Department of Anesthesiology and Intensive Therapy, Faculty of Medicine, Universitas Indonesia, Dr. Cipto Mangunkusumo Hospital, Diponegoro Street No. 71, Jakarta 10430, Indonesia, email: riyadh.firdaus@ui.ac.id Department of Anesthesiology and Intensive Therapy, Faculty of Medicine, Universitas Indonesia, Dr. Cipto Mangunkusumo Hospital, Jakarta 10430, Indonesia

O Open Access. ( 2021 Firdaus et al., published by Sciendo. (cc) B Attribution 4.0 License. 
[7]. To explore further the complex processes that occur in TBI in humans, several models of TBI have been developed using laboratory animals [6].

Propofol (2,6-bis(propan-2-yl)phenol) is an anesthetic agent, which is often used intravenously in the management of TBI, including for surgical intervention or sedation in an intensive care unit [8]. Propofol has advantages of minimal side effects and a short onset of drug activation and duration, along with relatively facile control of the depth of anesthesia. Apart from its anesthetic effects, propofol has properties as an anxiolytic, immunomodulator, analgesic, antiemetic, and inhibits platelet aggregation among other effects [9]. Propofol has shown neuroprotective effects due to its antioxidant activity and its ability to decrease brain metabolic rate, redistribute cerebral blood flow, suppress glutamates during ischemic events, and regulate apoptosis-related proteins [10].

Although there are many studies investigating the neuroprotective effect of propofol in TBI, other studies have produced discordant results. Propofol has shown neurotoxic effects on the brain, which caused increased mortality, worsened neurobehavioral outcomes, and reduced hippocampal neurogenesis [11]. To our knowledge, no systematic review has addressed this issue to date. Here, we sought to identify systematically studies from the literature to determine the effect of propofol in terms of physiological parameters, post administration effects, and dose used to determine the dominant effect of propofol in rodent models of TBI.

\section{Methods}

This systematic review was conducted according to the recommendations of the preferred reporting items for systematic reviews and meta-analysis with a PRISMA 2020 statement checklist [12]. The research question was "In studies in rodent models of TBI, does propofol exhibit neuroprotective effects?". This research question was constructed following the PICOS guidelines [13].

\section{Study selection}

We selected articles that met the inclusion criteria, which were a trial using a rodent model of TBI, with propofol as one of the intervention agents. We also included articles comparing the neuroprotective effects of propofol with other agents in rodent models of TBI. We excluded articles that were not available in full-text form and studies that were not published in English.

\section{Data sources and searches}

Relevant publications were identified using PubMed, EBSCO Host, and ProQuest search engines without restrictions or filters of publication date or language. The search keywords included MeSH: propofol, TBI, traumatic brain injury, neuroprotection, neuroprotective agents, and rodents. In addition to the MeSH keywords, we also added 2 other keywords not listed in the MeSH: neurotoxicity and craniocerebral trauma. A reference search from selected articles was conducted manually. All study searches were completed by March 1, 2021.

\section{Data extraction and result synthesis}

Data was extracted from the selected studies by 2 reviewers independently using Cochrane standardized data extraction form [14]. Data extracted from each study included year of publication, study design, study objectives, number of experimental groups, analytical methods, stated limitations and recommendations, and the study conclusions. A validity assessment was also conducted using the Cochrane risk-ofbias tool [15]. All data extracted from the selected studies were compiled into a table and explained qualitatively.

\section{Results}

\section{Study identification}

Based on the search strategy described above, 13,540 studies were found. After duplicates were removed, 9,286 studies remained, and prespecified criteria excluded 2,018 more studies, leaving 7,268. Then, these studies were screened by reading the titles and abstracts of each study, and 17 relevant studies were obtained. Of the 17 studies, we evaluated eligibility and feasibility of data extraction with a standardized form and found 12 that met the requirements for data extraction and further analysis (Figure 1).

\section{Selected study quality}

The assessment was conducted using the inclusion criteria. Overall, the study findings show a large variety of trauma types and methods used to evaluate the effects of propofol. The research methods and designs used were heterogeneous. Most of the researchers did not explain the bias that might occur in their respective studies [16-19]. Only 3 articles described 


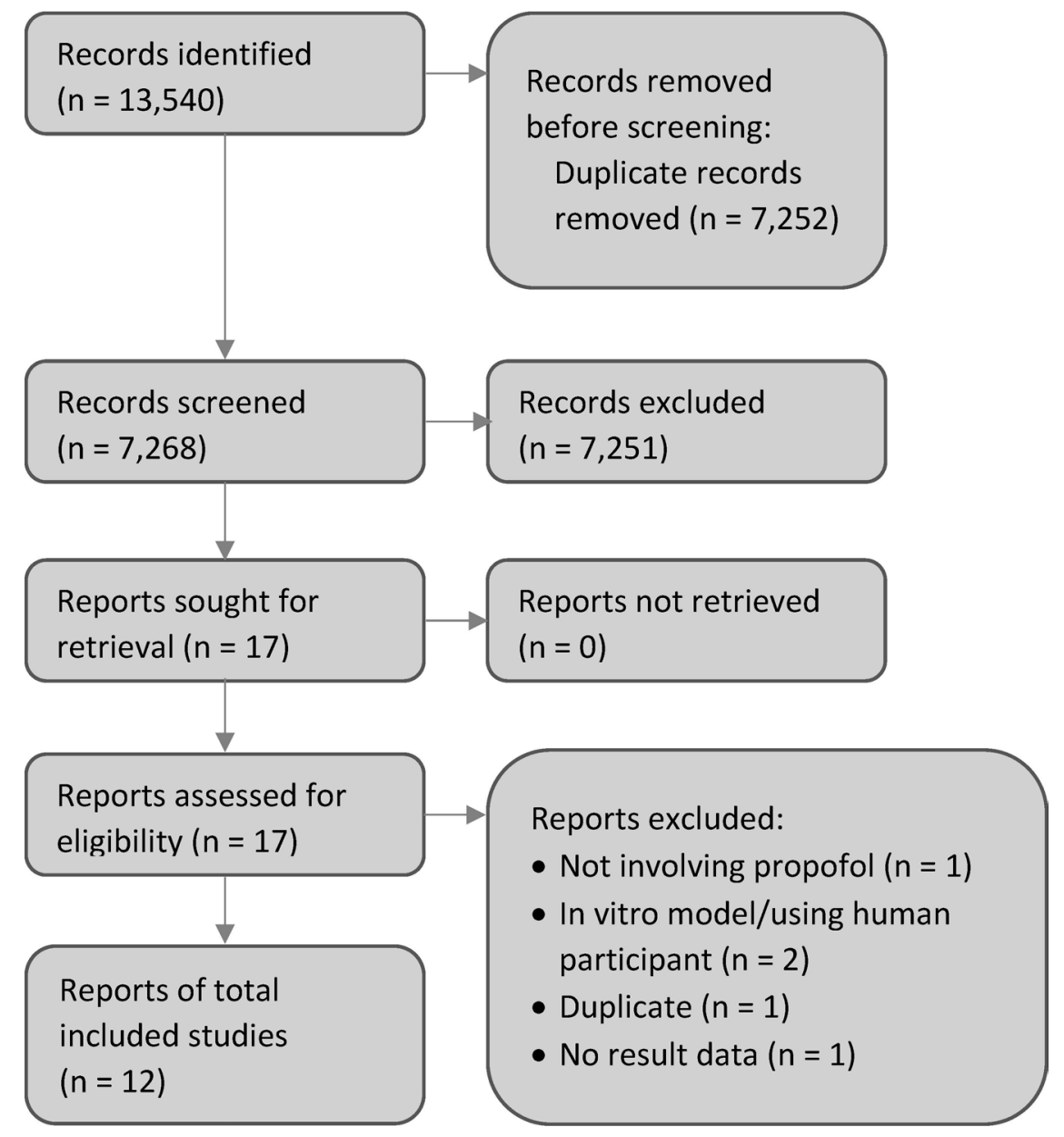

Figure 1. Flow diagram of the study selection process.

the bias that could affect the results of the study they reported [20-22]. Moreover, only one study used investigators blinded to the treatment during the intervention process and the assessment of intervention results [23].

\section{Main results}

\section{Physiological changes}

Characteristics of the included studies are shown in Table 1. Of the 12 studies included in the present research, 8 assessed the physiological parameters of experimental animals. Mean arterial pressure (MAP), heart rate, $\mathrm{pH}, \mathrm{PaCO}_{2}$, and $\mathrm{PaO}_{2}$ were not found to be significantly different in 4 studies, both in the pre- and post-trauma evaluation, and the evaluation in each experimental group. Another 3 articles did not state whether there was a change in physiological factors during the experiment, and the remaining article reported differences found in physiological parameters that were affected by the dose of propofol administered [16, 18-20, 22-26]. Apart from the previously mentioned physiological parameters, studies by Sebastiani et al. [23], Statler et al. [24], and Thal et al. [25] also assessed core temperature, brain tissue temperature, hematocrit, and serum glucose levels in rats or mice during the experimental procedure, all of which were within normal limits, and there were no differences found between groups.

\section{Effects of propofol administration on lesion volume}

A significant reduction in lesion volume after administration of propofol was found in 2 studies. The volume reduction effect is stronger when propofol is given early [17, 22]. No change in lesion size after administration of propofol was found in another 2 studies, even when low dose $(20 \mathrm{mg})$ and high dose $(30 \mathrm{mg}$ ) of propofol were administered to investigate a possible dose-dependent effect [16, 25]. Sebastiani et al. [23] found that early propofol administration ( $6 \mathrm{~h}$ post injury) did 


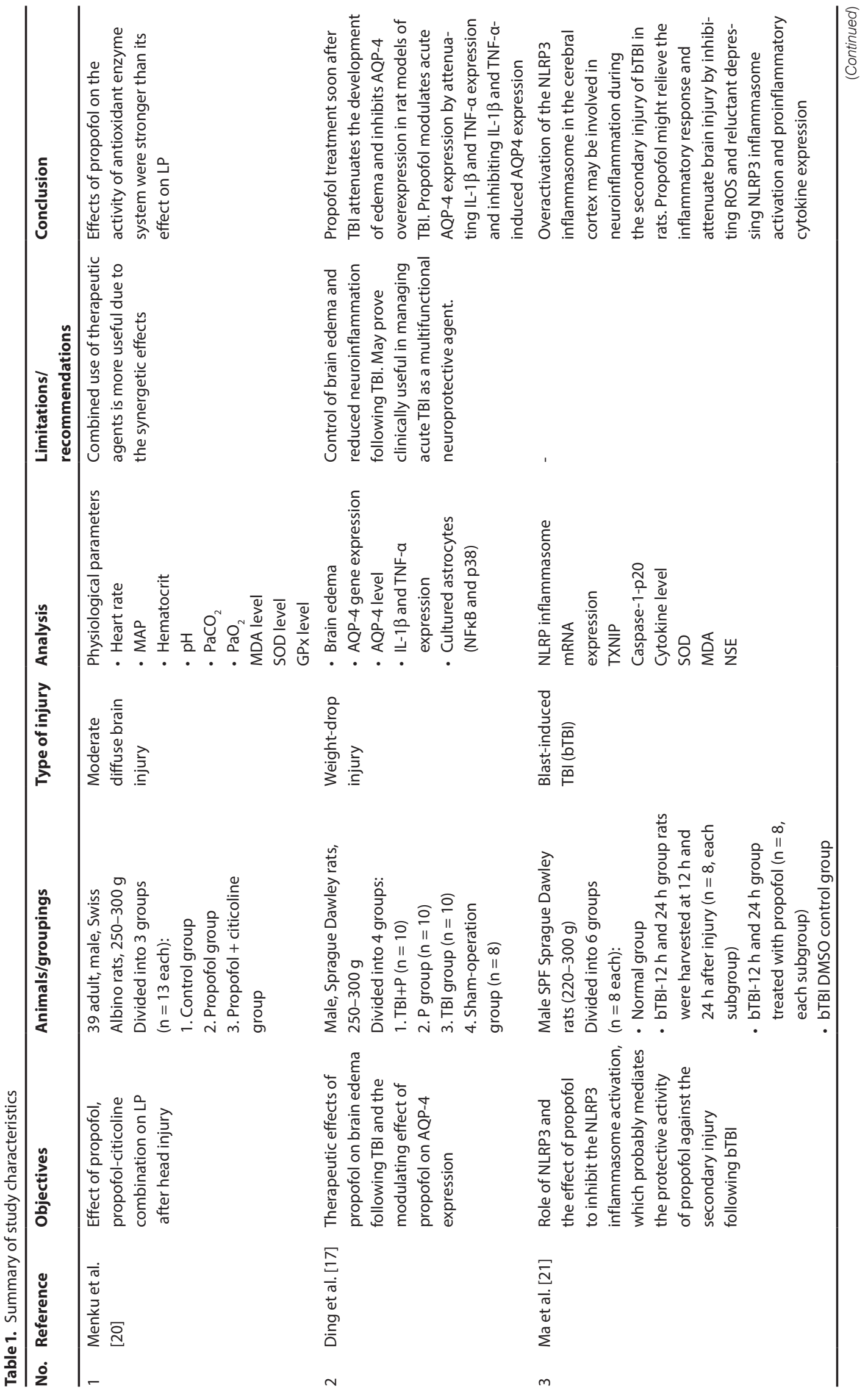




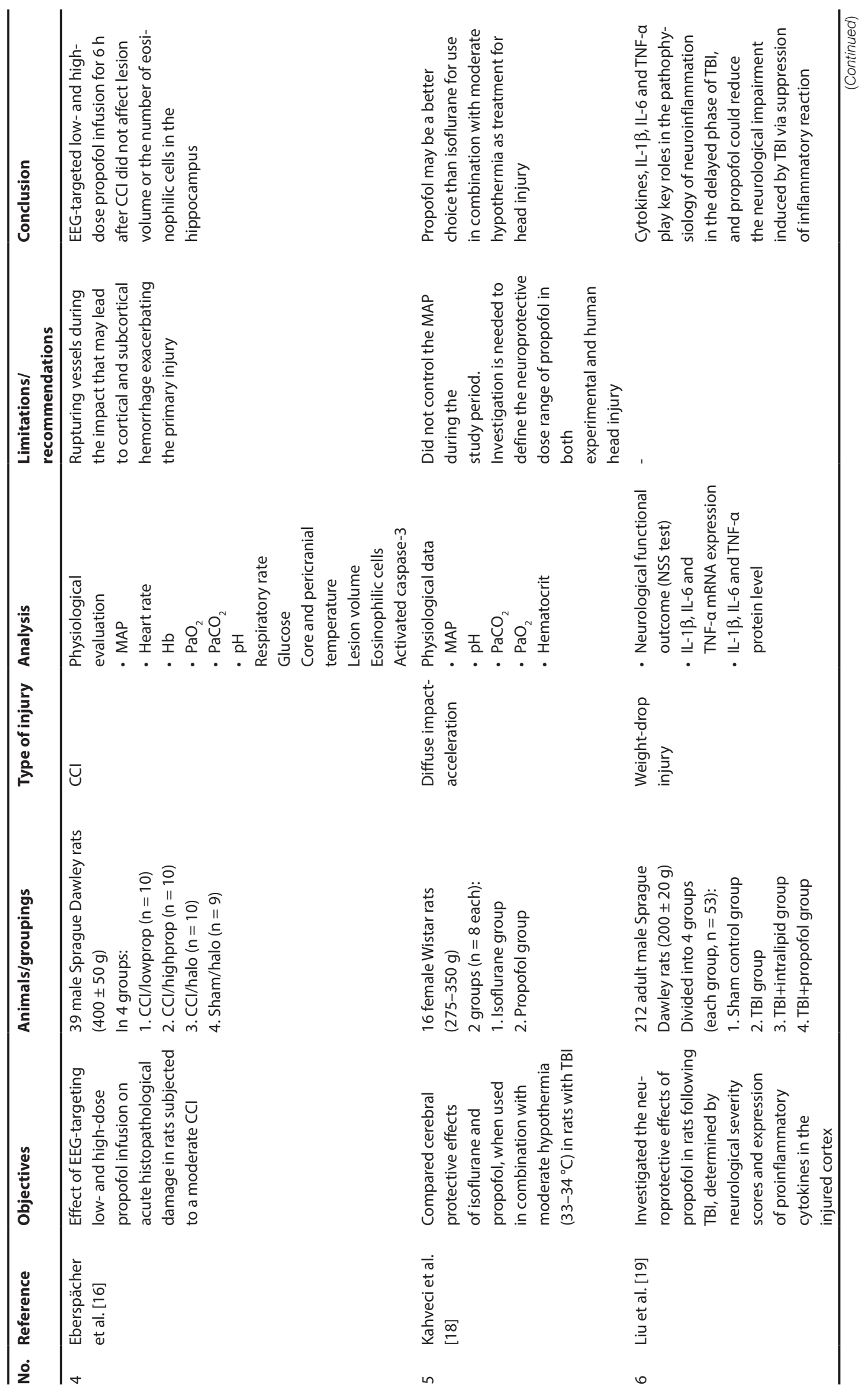




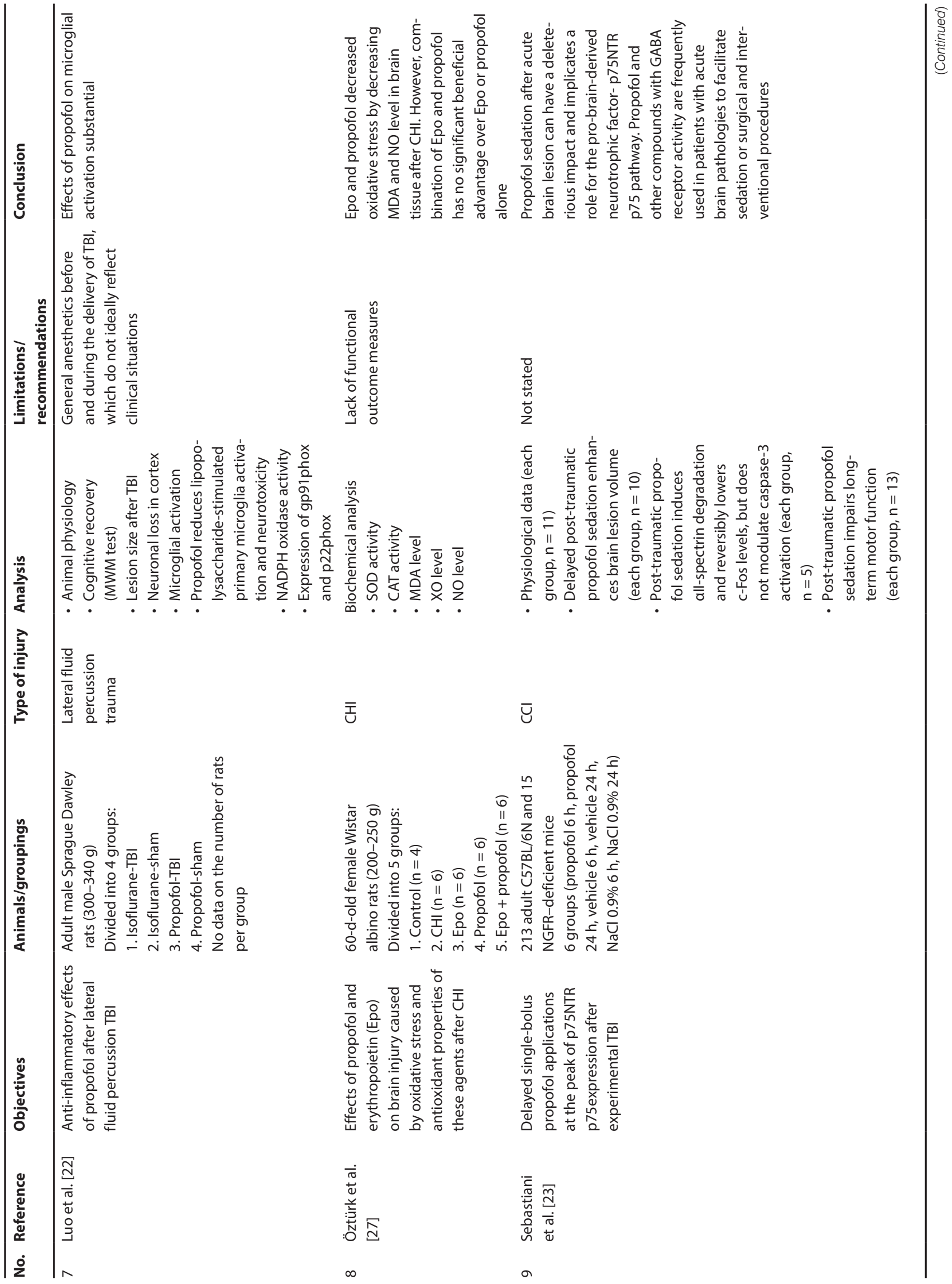




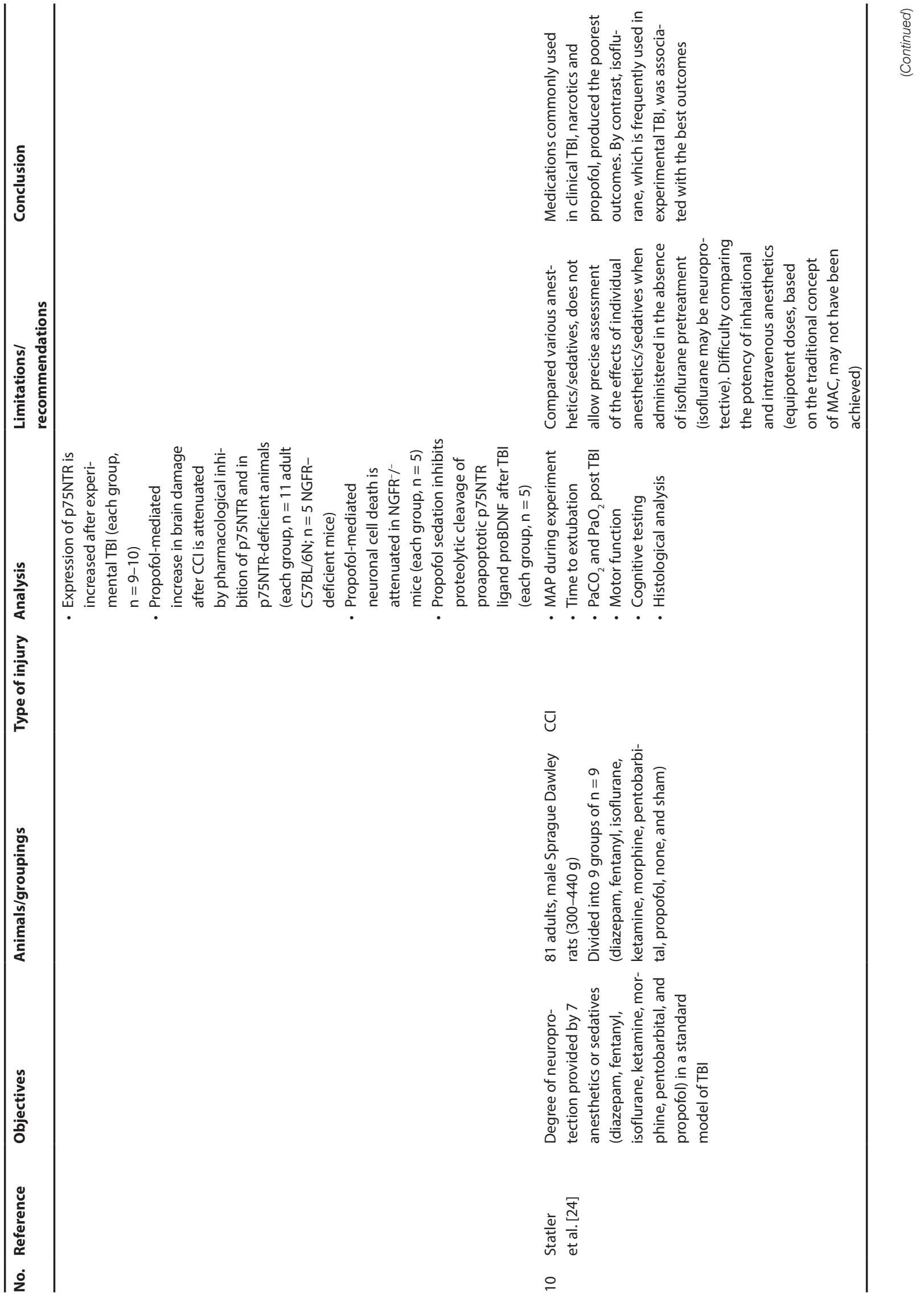




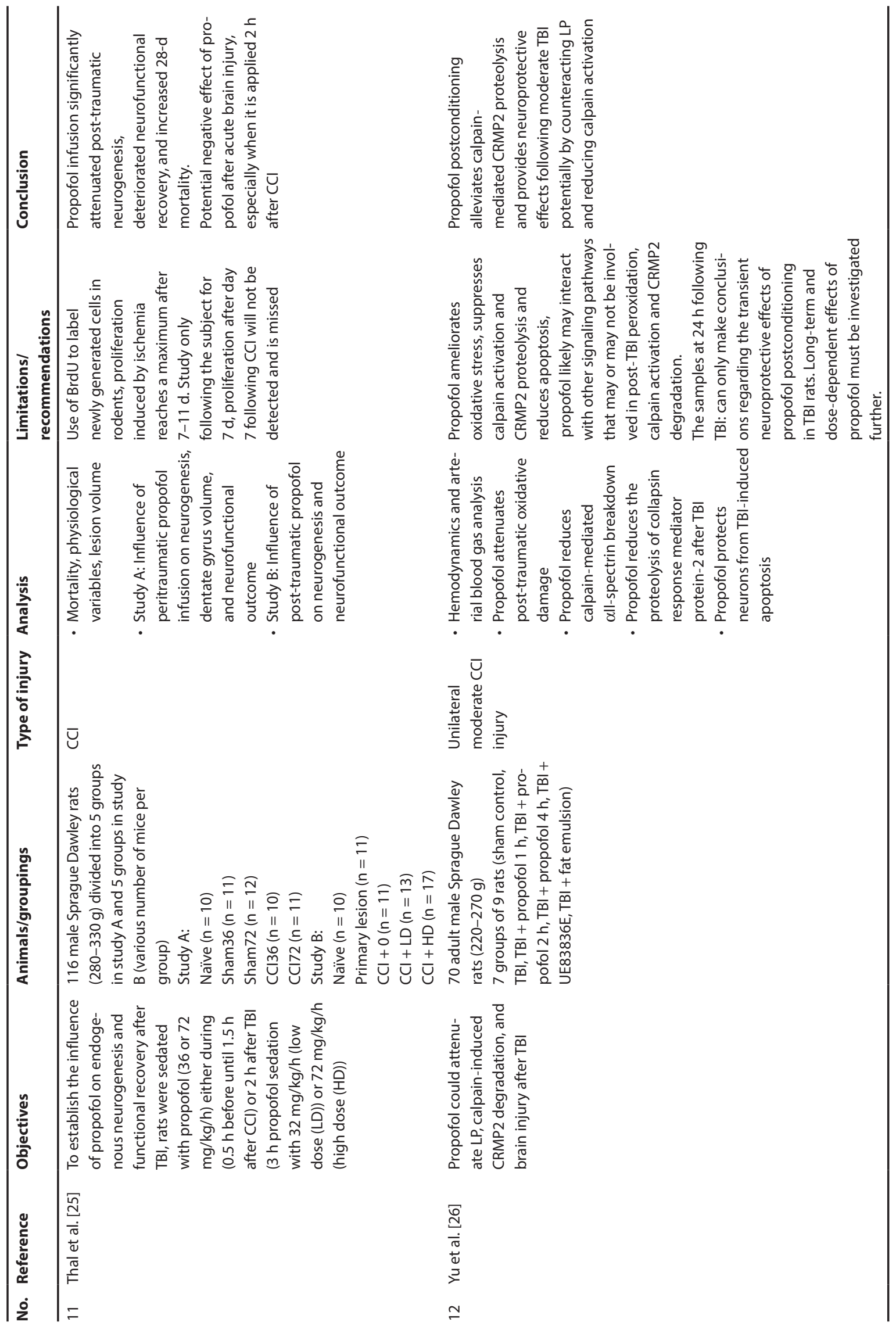




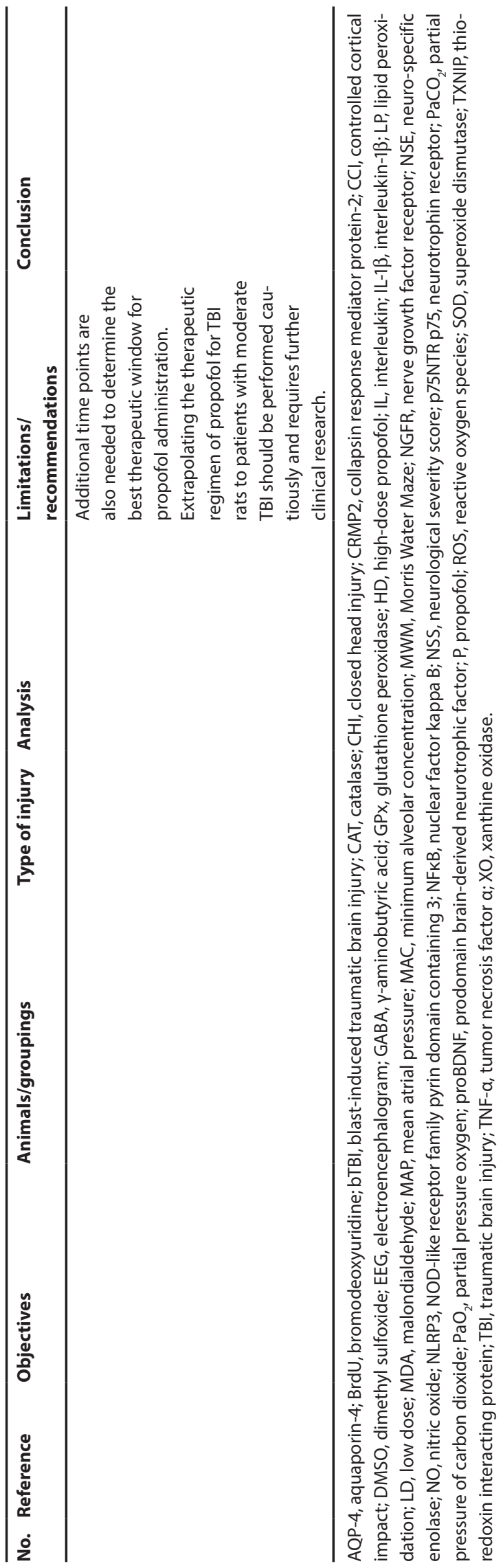

not substantially influence lesion volume. Meanwhile, delayed propofol administration ( $24 \mathrm{~h}$ after exposure to trauma) was associated with significant increase of lesion volume in a mouse group with propofol sedation compared with a control group. Statler et al. [24] found propofol increases lesion size in adult rats, as do other anesthetic agents. There was no lesion size data for the remaining 6 studies.

\section{Effects of propofol administration on antioxidant release}

From the studies analyzed, we found 3 that assessed the levels of superoxide dismutase (SOD) and malondialdehyde (MDA) in rats administered propofol and found an increase in SOD levels and a decrease in MDA levels in brain tissue [20, 21, 27]. However, Öztürk et al. [27] did not find a significant difference in SOD levels. In addition to SOD and MDA levels, Öztürk et al. [27] also measured the levels of xanthine oxidase (XO), levels of nitric oxide (NO), and catalase activity (CAT) in brain tissues. CAT levels were not found to be significantly different between the groups, while $\mathrm{XO}$ and $\mathrm{NO}$ levels were lower in the propofol group. The difference in NO levels was significant, while XO levels were not. Ma et al. [21] measured glutathione (GSH) levels, another indicator in assessing the oxidative stress response, which were elevated after propofol administration.

\section{Effect of propofol administration on the release of inflammatory factors}

Inflammatory factors such as interleukin-1 $\beta$ (IL-1 $1 \beta$ ) and tumor necrosis factor- $\alpha$ (TNF- $\alpha$ ) are released spontaneously in brain tissue after TBI, but propofol can reduce their levels. Ding et al. [17] specifically described the release of IL-1 $\beta$ and TNF- $\alpha$ and the effect of propofol on pyrrolidine dithiocarbamate (PDTC) activation pathway as an inhibitor of nuclear factor kappa B (NFKB) and SB203580 as an inhibitor of P38 mitogen-activated protein kinases (p38/MAPK), which was used to assess specific pathways of aquaporin-4 (AQP-4) expression. Propofol acts as an inhibitor of both the NFKB and p38/MAPK pathways. qRT-PCR analysis found that propofol clearly inhibits the expression of the AQP-4 mRNA in vivo, which is mediated by NFKB. This inhibition by propofol is influenced by the dose given and the time of administration. The explanation for the timing of administration effectiveness relates to activation of acute transcription of AQP-4 after the onset of TBI [17]. A decrease in the levels of the NOD-like receptor family pyrin domain containing 3 (NLRP3) inflammasome occurred after propofol administration in rats. The levels of IL- $1 \beta$ and TNF- $\alpha$ in the cerebral cortex tissue increased in the first $12 \mathrm{~h}$ and 
$24 \mathrm{~h}$ after trauma, while propofol administration at the time of trauma decreased release of these inflammatory factors [21]. After the administration of propofol, the expression of IL-1 $\beta$, IL- 6 , and TNF- $\alpha$ mRNA decreased inflammation in rats compared with an experimental group without propofol administration. After the administration of propofol, the protein levels of the 3 cytokines were significantly decreased [19]. Based on these 3 studies, we infer that propofol exerts neuroprotective effects by reducing inflammatory factors $[17,19,21]$.

\section{Neurotoxic effects of propofol}

Sebastiani et al. [23] found propofol had neurotoxic effects through calpain activity increase as seen from the quantification of $\alpha$ II-spectrin degradation results. Calpain or calciumregulated cysteine protease promotes cell death. The amount of $\alpha$ II-spectrin degradation was not affected by propofol in the first hour after trauma, but increased significantly in the following $6 \mathrm{~h}$. Moreover, propofol administration expanded lesions because of TBI via a neurotrophic receptor pathway by mediating an increase of pro-brain-derived neurotrophic factorp75 levels. Yu et al. [26] found propofol had the opposite effect. After propofol administration up to $4 \mathrm{~h}$ after TBI, the quantity of $\alpha$ II-spectrin degradation products was significantly lower in the pericontusional cortex of rats at $24 \mathrm{~h}$ after TBI compared with vehicle control, although we note the use of chloral hydrate in their model which is unacceptable for anesthesia.

\section{Effects of propofol on neurological function}

Neurological function has been assessed based on the motor and cognitive functions of rat models. Motor function in rats was assessed by beam balance and beam walking tests. The rats from both propofol-administered and control groups showed disturbances in motor function after injury. However, Statler et al. [24] showed that rats in a group given propofol showed more severe impairment than the control group. Thal et al. [25] also showed that motor impairment was more severe in rats administered larger doses $(72 \mathrm{mg} / \mathrm{kg} / \mathrm{h})$ of propofol. Cognitive function was assessed using the Morris Water Maze (MWM) and Barnes maze tests [24, 25]. In the MWM, rats in the propofol group showed slower learning ability than a control group [24]. The Barnes maze test results varied greatly between rats; and the investigators were unable to show any significant differences between groups [25]. Lou et al. [22] found no significant changes on MWM assessment on days 14 and 17 after TBI followed immediately by propofol administration.

\section{Propofol doses}

The doses of propofol administered in the various studies differed widely, as did the route of drug administration. In 5 studies, the dose range for bolus intraperitoneal administration of propofol ranged from $50 \mathrm{mg} / \mathrm{kg}$ to $100 \mathrm{mg} / \mathrm{kg}$ [17, 19-21, 27]. However, standard administration of propofol is by intravenous infusion. In another 5 studies, intravenous administration with doses varying from $10 \mathrm{mg} / \mathrm{kg} / \mathrm{h}$ to $85 \mathrm{mg} / \mathrm{kg} / \mathrm{h}$ were used. Yu et al. [26] used intravenous bolus. It is not clear which dose of propofol caused neuroprotective effects nor is it clear which dose causes neurotoxic effects [18, 23-26].

\section{Discussion}

The effects of propofol on brain tissue after TBI can be neuroprotective or neurotoxic and are not yet fully understood. Ethical considerations are a factor that makes the effects of propofol difficult to study directly in humans. In trials, it is not possible to observe directly the processes that occur in the patient's brain histologically, biochemically, or immunohistochemically. Although its effects can be neuroprotective or neurotoxic, propofol is an anesthetic agent commonly used in intensive care units because of its high safety profile. Moreover, propofol is superior to benzodiazepines or other drugs because it carries less risk of causing respiratory system suppression and has a short sedation duration, making it possible to carry out a periodic neurological assessment [28]. Therefore, we conducted a systematic review to investigate the physiological changes, the changes of the traumatic lesion size, and the neuroprotective or neurotoxic mechanisms arising from propofol administration to rodents. Rodents are commonly chosen for experimental models of TBI as they are affordable, small, and have standardized physiological parameters [29].

The neuroprotective effects of propofol in the rodent models of TBI are associated with antioxidants, potentiation of $\gamma$-aminobutyric acid type $\mathrm{A}\left(\mathrm{GABA}_{\mathrm{A}}\right)$ receptors, which mediate inhibition of synaptic transmission and inhibition of glutamate release. Propofol also modulates various aspects of the host inflammatory response. It reduces the release of proinflammatory cytokines, alters NO expression, interferes with the function of monocytes and neutrophils, and has the potential to counteract free radicals [8]. Moreover, propofol administration combined with moderate hypothermia can significantly reduce intracranial pressure and increase cerebral perfusion pressure. Administration of propofol before weight-drop injury demonstrated a neuroprotective effect in rats by modulating NO synthesis and reducing lipid peroxidation (LP) [30]. 
Propofol is commonly used in patients with acute brain injury to facilitate sedation during surgery. In the present study, we reviewed 12 studies in rodents to identify the neuroprotective or neurotoxic effects of propofol. We found 8 studies indicating propofol having neuroprotective effects through various mechanisms. We also found studies indicating neurotoxic effects from propofol administration, which were induction of neuron cell death through the neurotrophic receptor pathway by mediating pro-brain-derived neurotrophic factor-p75, deteriorating motor function, interfering with the neurogenesis process, and increased mortality.

\section{Physiological changes}

Propofol administration often causes side effects in the form of hypotension. Hypotension is categorized as a mild side effect when compared with the advantages of propofol which can maintain cerebral perfusion pressure in patients with TBI [28]. Propofol did not show any significant effect on the physiological parameters of rodents that underwent experimental TBI, either before or after the trauma $[16,18$, $20,22-26]$. These findings are consistent with data on propofol in humans, which shows a good safety profile [28, 31, 32]. Statler et al. [24] explored the duration from trauma to extubation, and found rats administered propofol required a significantly longer time to extubation than those administered isoflurane, rats in a sham group, and rats that did not receive additional anesthetic agents.

\section{Propofol effects}

Controlled cortical impact (CCI) type trauma in rodent models of TBI produced an increase in the volume of brain lesions after $24 \mathrm{~h}$ [33]. Histological assessment by Luo et al. [22] found a decrease in the volume of lesions after administration of propofol compared with that after administration of isoflurane. However, Eva et al. [16] found that the use of propofol did not affect lesion volume when compared with halothane as the anesthetic agent. The difference in the results might be a consequence of differences in the weight of the rats and the type of head injury. The overall primary injury lesion volume found by Thal et al. [25] was $23.8 \pm 7.4 \mathrm{~mm}^{3}$, although not all data were shown. This study found no significant differences in lesion volume associated with propofol administration. Propofol administration within $24 \mathrm{~h}$ post trauma has shown an effect of increasing the lesion volume. However, the administration of propofol within $6 \mathrm{~h}$ produced no significant increase of lesion volume [23].
Secondary injury in TBI results in many pathophysiological effects, such as ion imbalance, inflammation, apoptosis, endoplasmic reticulum stress, and oxidative stress [34]. Oxidative stress itself produces reactive oxygen species (ROS) and causes a redox imbalance, producing LP, nucleic acid oxidation, and DNA damage leading to neuronal damage and neuron cell death [35]. Lipid peroxidase activity is assessed by the level of MDA, and the changes in cerebral cortex endogenous antioxidants post TBI can be assessed based on levels of SOD and GSH. As expected, propofol administration increased the activity of SOD and GSH, which would decrease MDA levels in the process of oxidative stress [21]. In the present review, we found propofol administration reduced oxidative stress by attenuating microglia activation, MDA, and NO levels.

The inflammatory response after TBI will activate microglia and astrocytes, release inflammatory mediators in the brain, and mobilize peripheral immune cells (e.g., leukocytes and monocytes). Biomarkers commonly used for antiinflammatory and proinflammatory molecules are TNF- $\alpha$, IL-1 $\beta$, IL-6, IL-8, and IL-10 [36]. Propofol can decrease neurological damage by suppressing the inflammatory reaction, with the inflammatory cytokines most affected by propofol administration being IL- $1 \beta$ and TNF- $\alpha[17,19,21]$. Overactivation of the NLRP3 inflammasome after trauma in the cerebral cortex affects the neuroinflammatory processes leading to secondary trauma. Propofol can mitigate the inflammatory response, reduce brain damage by inhibiting ROS production and depress activation of the NLRP3 inflammasome, and influence the expression of proinflammatory cytokines [21]. The glymphatic system plays a role in directing cerebrospinal fluid to enter the brain through the periarterial space, into the interstitium via AQP-4. After TBI, the function of the glymphatic pathway is impaired and decreases by up to $60 \%$ [36]. Administration of propofol immediately after TBI reduces edema and will inhibit the overexpression of AQP-4 [17].

Apart from the direct effect of propofol on neurotransmission, propofol also results in impaired mitochondrial function in neurons, which is responsible for neurotoxicity and postoperative brain dysfunction. During the anesthesia, propofol inhibits electrophysiological processes that can inhibit ATP requirements [37]. Sebastiani et al. [23] demonstrated that immediate administration of propofol after acute injury produces an adverse effect mediated by pro-brain-derived neurotrophic factor-p75. By contrast, propofol also increases calpain activity as seen from the high degradation result of $\alpha$ II-spectrin by the calpain-dependent proteolysis mechanism. Increased degradation of $\alpha$ II-spectrin by calpain-dependent proteolysis is thought to be a mechanism for propofol-induced neurotoxicity in the developing brain that results in structural changes, changes in synaptic plasticity, and activation of the 
cell death process [23]. By contrast, Yu et al. [26] did not find a neurotoxic effect of propofol. The significantly lower level of $\alpha$ II-spectrin degradation in the propofol group suggested that propofol was active and neuroprotective in the calpain activation pathway in TBI.

A strong adverse effect of propofol administration is a decrease in the post-traumatic neurogenesis process, especially if it is administered within a few hours post trauma. The brain will undergo physiological changes to maintain and enhance neurogenesis during TBI, whereas the process will be hindered by propofol infusion. Neurogenesis has an important role in memory function. The inhibition of neurogenesis results in a decrease in cognitive and motor function in both humans and rats [24, 25]. Statler et al. [24] showed that rats given propofol experienced more severe impairment than a control group [24]. Thal et al. [25] also showed that motor impairment was more severe in rats receiving propofol in larger doses. Impaired cognitive function on MWM assessment as an indicator of hippocampal damage can be measured by counting the number of neurons in the region. Luo et al. [22] found no significant difference of propofol administration in improving cognitive abilities according to a MWM test, as consistent with the state of TBI, which induces neuron cell death.

We did not limit the present review by the type of TBI. Therefore, the conclusions drawn are the result of general analysis and are not limited to particular types of TBI. Moreover, some of the literature analyzed reported propofol administration before and during the trauma (not only after the trauma process occurred). This may have biased conclusions on assessing the effects of propofol, which is most commonly used after trauma occurs.

\section{Conclusion}

Propofol has protective effects in rodent models of TBI via several mechanisms, including through $\mathrm{GABA}_{\mathrm{A}}$ receptormediated inhibition of synaptic transmission, decreased inflammatory reaction, which delays neurological damage, decreases oxidative stress, and inhibits glutamate release. Various doses have been used in research models of TBI to determine the effect of propofol; however, the doses required to obtain neuroprotective and neurotoxic effects are not yet clear. The timing of propofol administration after TBI, the dose of propofol administered, and the duration of propofol administration contribute to determining the effect of propofol on TBI. Further research is needed to determine the dose range effecting neuroprotection and neurotoxicity to guide clinical research in humans.
Author contributions. All authors contributed to the concept of the study. RF and RT conducted the literature review, data extraction, and risk of bias assessment. ST and RT analyzed and interpreted the data collected. RA and RT drafted the original manuscript. All authors critically revised the manuscript for important intellectual content, approved the final version of the manuscript, and agreed to be accountable for all aspects of the work and take responsibility for statements made in the published article.

Acknowledgments. The authors thank Universitas Indonesia for facilitating the research.

Conflicts of interest statement. The authors have each completed an International Committee of Medical Journal Editors Uniform Disclosure Form for Potential Conflicts of Interest. None of the authors declare any potential or actual conflict interest in relation to the present work.

Data sharing statement. The present review is based on the references cited. Further details and interpretation are available from the corresponding author on reasonable request.

\section{References}

[1] Carbonara M, Fossi F, Zoerle T, Ortolano F, Moro F, Pischiutta F, et al. Neuroprotection in traumatic brain injury: mesenchymal stromal cells can potentially overcome some limitations of previous clinical trials. Front Neurol. 2018; 9:885. doi: 10.3389/fneur.2018.00885

[2] James SL, Theadom A, Ellenbogen RG, Bannick MS, MontjoyVenning W, Lucchesi LR, et al.; GBD 2016 Traumatic Brain Injury and Spinal Cord Injury Collaborators. Global, regional, and national burden of traumatic brain injury and spinal cord injury, 1990-2016: a systematic analysis for the Global Burden of Disease Study 2016. Lancet Neurol. 2019; 18:56-87.

[3] Dewan MC, Rattani A, Gupta S, Baticulon RE, Hung Y-C, Punchak M, et al. Estimating the global incidence of traumatic brain injury. J Neurosurg. 2021; 130:1080-97.

[4] Centers for Disease Control and Prevention. Traumatic brain injury \& concussion [Internet]. Atlanta, GA: U.S. CDC; 2021 [cited 2021 Aug 5]. Available from: https://www.cdc.gov/traumaticbraininjury/get_the_facts.html

[5] Pitt J, Pitt Y, Lockwich J. Clinical and cellular aspects of traumatic brain injury. Chapter 44. In: Gupta RC, editor. Handbook of toxicology of chemical warfare agents. 3rd ed. Cambridge, MA: Academic Press; 2020, p. 745-66.

[6] Galgano M, Toshkezi G, Qiu X, Russell T, Chin L, Zhao L-R. Traumatic brain injury: current treatment strategies and future endeavors. Cell Transplant. 2017; 26:1118-30.

[7] Lazaridis C, Rusin CG, Robertson CS. Secondary brain injury: predicting and preventing insults. Neuropharmacology. 2019; 145:145-52. 
[8] Vanlersberghe C, Camu F. Propofol. In: Part III. Modern intravenous anesthetics. Camu F, section editor. In: Schüttler J., Schwilden H, editors. Modern Anesthetics. Berlin: Springer; 2008, p. 227-52. (Starke K, series editor-in-chief, Handbook of Experimental Pharmacology, vol. 182).

[9] Vasileiou I, Xanthos T, Koudouna E, Perrea D, Klonaris C, Katsargyris A, Papadimitriou L. Propofol: a review of its non-anaesthetic effects. Eur J Pharmacol. 2009; 605:1-8.

[10] Song C-Y, Xi H-J, Yang L, Qu L-H, Zhou J, Cui X-G, et al. Propofol inhibited the delayed rectifier potassium current $\left(I_{\mathrm{k}}\right)$ via activation of protein kinase $\mathrm{C}$ epsilon in rat parietal cortical neurons. Eur J Pharmacol. 2011; 653:16-20.

[11] Woldegerima N, Rosenblatt K, Mintz CD. Neurotoxic properties of propofol sedation following traumatic brain injury. Crit Care Med. 2016; 44:455-6.

[12] Page MJ, McKenzie JE, Bossuyt PM, Boutron I, Hoffmann TC, Mulrow CD, et al. The PRISMA 2020 statement: an updated guideline for reporting systematic reviews. Int J Surg. 2021; 88:105906. doi: 10.1016/j.ijsu.2021.105906

[13] Booth A, O’Rourke AJ, Ford NJ. Structuring the pre-search reference interview: a useful technique for handling clinical questions. Bull Med Libr Assoc. 2000; 88:239-46.

[14] Higgins JPT, Thomas J, Chandler J, Cumpston M, Li T, Page MJ, Welch VA, editors. Cochrane handbook for systematic reviews of interventions. 2nd ed. Hoboken, NJ: Wiley Blackwell; 2019. pp. 694. doi: 10.1002/9781119536604

[15] Sterne JAC, Savović J, Page MJ, Elbers RG, Blencowe NS, Boutron I, et al. RoB 2: a revised tool for assessing risk of bias in randomised trials. BMJ 2019; 366: 14898. doi: 10.1136/bmj.14898

[16] Eberspächer E, Heimann K, Hollweck R, Werner C, Schneider G, Engelhard K. The effect of electroencephalogram-targeted highand low-dose propofol infusion on histopathological damage after traumatic brain injury in the rat. Anesth Analg. 2006; 103:1527-33.

[17] Ding Z, Zhang J, Xu J, Sheng G, Huang G. Propofol administration modulates AQP-4 expression and brain edema after traumatic brain injury. Cell Biochem Biophys. 2013; 67:615-22.

[18] Kahveci FS, Kahveci N, Alkan T, Goren B, Korfali E, Ozluk K. Propofol versus isoflurane anesthesia under hypothermic conditions: effects on intracranial pressure and local cerebral blood flow after diffuse traumatic brain. Surg Neurol. 2001; 56:206-14.

[19] Liu F, Chen M-R, Liu J, Zou Y, Wang T-Y, Zuo YX, Wang T-H. Propofol administration improves neurological function associated with inhibition of pro-inflammatory cytokines in adult rats after traumatic brain injury. Neuropeptides. 2016; 58:1-6.

[20] Menku A, Ogden M, Saraymen R. The protective effects of propofol and citicoline combination in experimental head injury in rats. Turk Neurosurg. 2010; 20:57-62.

[21] Ma J, Xiao W, Wang J, Wu J, Ren J, Hou J, et al. Propofol inhibits NLRP3 inflammasome and attenuates blast-induced traumatic brain injury in rats. Inflammation. 2016; 39:2094-103.

[22] Luo T, Wu J, Kabadi SV, Sabirzhanov B, Guanciale K, Hanscom M, et al. Propofol limits microglial activation after experimental brain trauma through inhibition of nicotinamide adenine dinucleotide phosphate oxidase. Anesthesiology. 2013; 119:1370-88.
[23] Sebastiani A, Granold M, Ditter A, Sebastiani P, Gölz C, Pöttker $B$, et al. Posttraumatic propofol neurotoxicity is mediated via the pro-brain-derived neurotrophic factor-p75 neurotrophin receptor pathway in adult mice. Crit Care Med. 2016; 44:e70-82.

[24] Statler KD, Alexander H, Vagni V, Dixon CE, Clark RSB, Jenkins L, Kochanek PM. Comparison of seven anesthetic agents on outcome after experimental traumatic brain injury in adult, male rats. Neurotrauma. 2006; 23:97-108.

[25] Thal SC, Timaru-Kast R, Wilde F, Merk P, Johnson F, Frauenknecht K, et al. Propofol impairs neurogenesis and neurologic recovery and increases mortality rate in adult rats after traumatic brain injury. Crit Care Med. 2014; 42:129-41.

[26] Yu Y, Jian M-Y, Wang Y-Z, Han R-Q. Propofol ameliorates calpain-induced collapsin response mediator protein-2 proteolysis in traumatic brain injury in rats. Chin Med J (Engl). 2015; 128:919-27.

[27] Öztürk E, Demirbilek S, Köroğlu A, But A, Begeç ZÖ, Gülec M, et al. Propofol and erythropoietin antioxidant properties in rat brain injured tissue. Prog Neuropsychopharmacol Biol Psychiatry. 2008; 32:81-6.

[28] Hausburg MA, Banton KL, Roman PE, Salgado F, Baek P, Waxman $\mathrm{MJ}$, et al. Effects of propofol on ischemia-reperfusion and traumatic brain injury. J Crit Care. 2020; 56:281-7.

[29] Xiong Y, Mahmood A, Chopp M. Animal models of traumatic brain injury. Nat Rev Neurosci. 2013; 14:128-42.

[30] Rowe RK, Harrison JL, Thomas TC, Pauly JR, Adelson PD, Lifshitz J. Using anesthetics and analgesics in experimental traumatic brain injury. Lab Anim (NY). 2013; 42:286-91.

[31] Marik PE. Propofol: therapeutic indications and side-effects. Curr Pharm Des. 2004; 10: 3639-49.

[32] Gu J-w, Yang T, Kuang Y-q, Huang H-d, Kong B, Shu H-f, et al. Comparison of the safety and efficacy of propofol with midazolam for sedation of patients with severe traumatic brain injury: a meta-analysis. J Crit Care. 2014; 29:287-90.

[33] Niesman IR, Schilling JM, Shapiro LA, Kellerhals SE, Bonds JA, Kleschevnikov AM, et al. Traumatic brain injury enhances neuroinflammation and lesion volume in caveolin deficient mice. J Neuroinflammation. 2014; 11:39. doi: 10.1186/1742-2094-11-39

[34] Chandran R, Kim T, Mehta SL, Udho E, Chanana V, Cengiz P, et al. A combination antioxidant therapy to inhibit NOX2 and activate Nrf2 decreases secondary brain damage and improves functional recovery after traumatic brain injury. J Cereb Blood Flow Metab. 2018; 38:1818-27.

[35] Zhou J, Wang H, Shen R, Fang J, Yang Y, Dai W, et al. Mitochondrial-targeted antioxidant MitoQ provides neuroprotection and reduces neuronal apoptosis in experimental traumatic brain injury possibly via the Nrf2-ARE pathway. Am J Transl Res. 2018; 10:1887-99.

[36] Xiong Y, Mahmood A, Chopp M. Current understanding of neuroinflammation after traumatic brain injury and cell-based therapeutic opportunities. Chin J Traumatol. 2018; 21:137-51.

[37] Berndt N, Rösner J, Haq RU, Kann O, Kovács R, Hermann K, et al. Possible neurotoxicity of the anesthetic propofol: evidence for the inhibition of complex II of the respiratory chain in area CA3 of rat hippocampal slices. Arch Toxicol. 2018; 92:3191-205. 